\title{
NOTE
}

\section{Amylose Selectively Includes a Specific Range of Molecular Weights in Poly(tetrahydrofuran)s in Vine-Twining Polymerization}

\author{
By Yoshiro KANEKO, Koutarou BEPPU, and Jun-ichi KADOKAWA*
}

KEY WORDS: Amylose / Enzymatic Polymerization / Inclusion Complex / Poly(tetrahydrofuran) / Selective Inclusion / Vine-Twining Polymerization /

Because specific inclusion to appropriate guest compounds is often appeared by host molecules, precise recognition of congeners, homologues, and other analogous molecules has been achieved by ability in inclusion of the host compounds. For example, crown ethers have respective cavities to fit sizes of congenerous cations, in which 18-crown-6, 15-crown-5, and 12-crown-4 have high affinities for potassium, sodium, and lithium cations, respectively, to favorably form inclusion complexes in each appropriate combination. ${ }^{1}$ Cyclodextrins, cyclic oligosaccharides consisting of 6-8 glucose units, also exhibit different inclusion abilities to some polyethers depending on whether their cavities fit sizes of the cross-sectional areas in the polymeric chains. ${ }^{2}$ On the other hand, amylose, a helical host compound that is described as follows in detail, shows the different inclusion ability to lipid homologues, forming inclusion complexes with them having appropriate alkyl chain lengths. ${ }^{3}$

Amylose is a polysaccharide with helical conformation linked through $(1 \rightarrow 4)-\alpha$-glycosidic linkages and also a wellknown host compound that forms inclusion complexes with slender guest compounds having relatively lower molecular weight by noncovalent interaction between guest molecules and the cavity of amylose. ${ }^{4}$ However, little has been reported regarding the formation of inclusion complexes between amylose and polymeric compounds. ${ }^{5}$ The main difficulty in incorporating polymeric compounds into the cavity of amylose is that the driving force for the binding is only caused by hydrophobic interactions. Amylose, therefore, does not have sufficient ability to include the long chains of guest polymers into its cavity.

By means of the enzymatic method for direct construction of the polysaccharides, ${ }^{6}$ we have developed a new methodology for the preparation of inclusion complexes composed of amylose and synthetic polymers, ${ }^{7}$ which was achieved by the enzymatic polymerization forming amylose in the presence of guest polymers. The representation of this reaction system is similar to the way that vines of plants grow twining around a rod. Accordingly, we have proposed that this polymerization method for the preparation of amylose-polymer inclusion complexes is named "vine-twining polymerization."
So far, we have found that appropriately hydrophobic polyethers, ${ }^{7 \mathrm{a}, \mathrm{c}}$ polyesters, ${ }^{7 \mathrm{~b}, \mathrm{~d}, \mathrm{i}}$ a poly(ester-ether) ${ }^{7 \mathrm{~d}}$ and polycarbonates $^{\text {th }}$ are included in the cavity of amylose by this polymerization system. For example, when the phosphorylasecatalyzed enzymatic polymerization was carried out in the presence of the aliphatic polyethers with appropriate hydrophobicity such as poly(oxetane) (POXT, - $\left[\mathrm{CH}_{2} \mathrm{CH}_{2} \mathrm{CH}_{2} \mathrm{O}\right]_{n}-$ ) and poly(tetrahydrofuran) (PTHF, $-\left[\mathrm{CH}_{2} \mathrm{CH}_{2} \mathrm{CH}_{2} \mathrm{CH}_{2} \mathrm{O}\right]_{n^{-}}$), these were included in the cavity of amylose to form the corresponding inclusion complexes. ${ }^{7 \mathrm{c}}$ However, in addition to no formation of the inclusion complex from poly(ethylene glycol) $\left(-\left[\mathrm{CH}_{2} \mathrm{CH}_{2} \mathrm{O}\right]_{n}-\right)$ because of its hydrophilic nature, the preparation of the inclusion complexes has not been achieved from the polyether with strong hydrophobicity like poly(oxepane) (- $\left.\left[\mathrm{CH}_{2} \mathrm{CH}_{2} \mathrm{CH}_{2} \mathrm{CH}_{2} \mathrm{CH}_{2} \mathrm{CH}_{2} \mathrm{O}\right]_{n^{-}}\right)$, attributed to its aggregation in the aqueous buffer of the solvent for the enzymatic polymerization.

These results have inspired us to achieve the selective inclusion toward two resemblant polymers in the vine-twining polymerization by means of slight difference in their hydrophobicities. In the previous papers, we reported that amylose selectively included one side of the polyethers, i.e., PTHF from a mixture of POXT/PTHF and one side of the polyesters, i.e., $\operatorname{poly}(\delta$-valerolactone $)$ from a mixture of $\operatorname{poly}(\delta$-valerolactone $) / \operatorname{poly}(\varepsilon$-caprolactone) by the vine-twining polymerization, respectively. ${ }^{7 \mathrm{~g}, \mathrm{j}}$ The difference in the chemical structures between the polymers in each mixture is only one methylene unit, and those having similar molecular weights were used. Therefore, this system is considered as a tool for discrimination of the polymers by means of the cavity of amylose.

On the basis of the previous studies and above findings, we have continuously investigated the selective inclusion to a specific range of molecular weights (MWs) in synthetic polymers by amylose in the vine-twining polymerization because synthetic polymers generally have molecular weight distribution (MWD), meaning that they are reasonably considered as mixtures of analogous molecules with different numbers of the repeating units. Additionally, the number of repeating unit (MW) contributes to show different properties of 
the polymer with the same structure of repeating unit. For example, PTHF with considerably low MW is soluble in water although PTHF is known as hydrophobic polymer, indicating that the hydrophobicity of PTHF is probably affected by its MW. These considerations have motivated us to attempt the selective inclusion to a specific range of MWs in PTHFs as guest polymers by amylose because we have already achieved the aforementioned selective inclusion of amylose toward two resemblant guest polymers. ${ }^{7 \mathrm{~g}, \mathrm{j}}$

In this study, we found that amylose selectively included a specific range of MWs in any PTHFs when the vine-twining polymerization was performed in the presence of some PTHFs with different average MWs.

\section{EXPERIMENTAL}

\section{Materials}

Phosphorylase ( $c a .300$ units/mL) was supplied from Ezaki Glico Co. Ltd., Osaka, Japan. ${ }^{8}$ PTHFs were prepared by ringopening polymerization of tetrahydrofuran according to the experimental method reported in previous literature. ${ }^{9}$ In the present study, three PTHFs with different MWs were prepared; PTHF-1K $\left(M_{\mathrm{n}}\right.$ and $M_{\mathrm{w}} / M_{\mathrm{n}}$ were estimated by ${ }^{1} \mathrm{H}$ NMR and gel permeation chromatography (GPC) measurements to be 1350 and 2.86, respectively), PTHF-3K $\left(M_{\mathrm{n}}\right.$ and $M_{\mathrm{w}} / M_{\mathrm{n}}$ were estimated by ${ }^{1} \mathrm{H}$ NMR and GPC measurements to be 3040 and 3.13, respectively), and PTHF-6K $\left(M_{\mathrm{n}}\right.$ and $M_{\mathrm{w}} / M_{\mathrm{n}}$ were estimated by ${ }^{1} \mathrm{H}$ NMR and GPC measurements to be 6330 and 2.45 , respectively). Maltoheptaose $\left(\mathrm{G}_{7}\right)$ was prepared by selective cleavage of one glycosidic bond of $\beta$-cyclodextrin under acidic condition. ${ }^{10}$ Other reagents and solvents were used without further purification.

\section{Typical Procedures for the Preparation of Amylose-PTHF Inclusion Complexes by Vine-Twining Polymerization}

PTHF-1K (150 mg) was suspended in sodium acetate buffer ( $4.0 \mathrm{~mL}, 0.2 \mathrm{~mol} / \mathrm{L}, \mathrm{pH}=6.2$ ) using an ultrasonic wave. After the addition of $\mathrm{G}_{7}$ primer $(6.9 \mathrm{mg}=6.0 \mu \mathrm{mol})$ and $\alpha$-D-glucose 1-phosphate disodium salt (G-1-P, $456.3 \mathrm{mg}=1.5 \mathrm{mmol})$, adjustment of the $\mathrm{pH}$ value to 6.2 using acetic acid aqueous solution $(0.20 \mathrm{~mol} / \mathrm{L})$ was carried out. Phosphorylase $(80 \mu \mathrm{L}=$ 24 units) was added to this solution, and then the solution was stirred vigorously at $40-45^{\circ} \mathrm{C}$ for $6 \mathrm{~h}$. The precipitated product was collected by filtration, washed with acetone and water, and dried under reduced pressure at room temperature to yield $c a$. $72.8 \mathrm{mg}$ of the inclusion complex. ${ }^{1} \mathrm{H} \mathrm{NMR}(400 \mathrm{MHz}$, DMSO- $d_{6}$ ): $\delta$ 1.40-1.60 (br, $-\mathrm{CH}_{2} \mathrm{CH}_{2} \mathrm{O}-$ due to PTHF), $\delta$ 3.10-3.92 (m, $\mathbf{H}_{2}-\mathbf{H}_{6}$ due to amylose, $-\mathrm{CH}_{2} \mathrm{O}$ - due to PTHF overlapping with HOD), $\delta 4.59,5.42,5.52(\mathrm{OH}), \delta 5.10$ (br, $\mathbf{H}_{\mathbf{1}}$ due to amylose). The degree of polymerization (DP) values of amylose in the resulting inclusion complexes were calculated by the integrated ratios of the signal of $\mathbf{H}_{1}$ of amylose chain $(\delta$ 5.1) to the signals of $\mathbf{H}_{1}$ ( $\alpha$ and $\beta$ ) of the reducing terminus ( $\delta$ $4.9(\alpha)$ and $4.3(\beta))$ in the ${ }^{1} \mathrm{H}$ NMR spectra (DMSO- $d_{6}-\mathrm{D}_{2} \mathrm{O}$ ). The DP values were assessed to be $c a$. 69, corresponding to $M_{\mathrm{n}}=c a$. 11200 .

\section{Typical Procedures for the Extraction of PTHFs from the Inclusion Complexes}

The inclusion complex $(30.0 \mathrm{mg})$ prepared by the aforementioned vine-twining polymerization was dissolved in DMSO $(0.20 \mathrm{~mL})$. After the solution was stirred at $40^{\circ} \mathrm{C}$ for $13 \mathrm{~h}$, it was added to excess amount of acetone. After the resulting precipitate was removed by filtration, the filtrate was evaporated. Then, chloroform $(10 \mathrm{~mL})$ and water $(10 \mathrm{~mL})$ were added to the resulting product solution in DMSO and the chloroform phase was separated, which was washed with water, evaporated, and dried under reduced pressure to give PTHF (2.7 mg).

\section{Measurements}

The ${ }^{1} \mathrm{H}$ NMR spectra were recorded using a JEOL ECX400 spectrometer (JEOL Ltd.). The X-ray diffraction (XRD) measurements were performed at a scanning speed of $2 \theta=$ $2^{\circ} /$ min using a Geigerflex RAD-IIB diffractometer (Rigaku Co.) with $\mathrm{Ni}$-filtered $\mathrm{CuK} \alpha$ radiation $(=0.15418 \mathrm{~nm})$. The GPC analyses were performed by using a HITACHI pump L2130 and a HITACHI RI detector L-2490 under the following conditions: Shodex GPC KF-804L and KF-803L columns with chloroform as the eluent at a flow rate of $1.0 \mathrm{~mL} / \mathrm{min}$ at $40{ }^{\circ} \mathrm{C}$.

\section{RESULTS AND DISCUSSION}

The vine-twining polymerization was performed by the phosphorylase-catalyzed enzymatic polymerization of G-1-P from $\mathrm{G}_{7}$ as a primer (feed molar ratio of $\mathrm{G}-1-\mathrm{P} / \mathrm{G}_{7}=250$ ) in the presence of PTHF-1K (a), PTHF-3K (b), or PTHF-6K (c) as the guest polymer in sodium acetate buffer $(0.2 \mathrm{~mol} / \mathrm{L}$, $\mathrm{pH}=6.2$ ) at $40-45^{\circ} \mathrm{C}$ for $6 \mathrm{~h}$, respectively (Scheme 1 ). The DPs of the enzymatically synthesized amylose by phosphorylase are generally changed by the feed molar ratios of G-1-P/ $\mathrm{G}_{7}$. The present polymerization system in lower feed molar ratio of $\mathrm{G}-1-\mathrm{P} / \mathrm{G}_{7}$, e.g., 10 , did not yield the inclusion complex because of the production of amylose with considerably low DP, which had no helical structure. On the other hand, when the polymerization system was performed in higher feed molar ratio of $\mathrm{G}-1-\mathrm{P} / \mathrm{G}_{7}$ such as 1000 , the $\mathrm{DP}$ of amylose was not higher than $c a$. 75. In the vine-twining polymerization, the enzymatic polymerization proceeds with forming the inclusion complex composed of amylose with V-type crystalline structure, which leads to precipitation of the product during the polymerization. The precipitation of the inclusion complex causes a difficulty in increasing the DP of amylose by the vinetwining polymerization even when the higher feed molar ratio of $\mathrm{G}-1-\mathrm{P} / \mathrm{G}_{7}$ is employed. On the basis of these results and our previous experiences, we consider that the favorable feed molar ratio of $\mathrm{G}-1-\mathrm{P} / \mathrm{G}_{7}$ for the vine-twining polymerization in this study is $c a$. 250. The precipitated products were collected and characterized by the following ${ }^{1} \mathrm{H}$ NMR and XRD measurements to be the inclusion complexes as shown in our previous papers. $^{7 \mathrm{a}, \mathrm{c}}$

The ${ }^{1} \mathrm{H}$ NMR spectrum in DMSO- $d_{6}$ of the product obtained by the vine-twining polymerization using PTHF-1K showed 


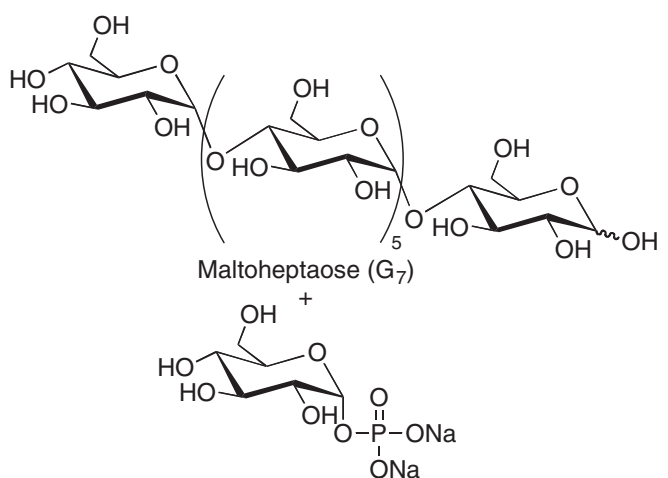

$\alpha$-D-Glucose 1-phosphate

$(\mathrm{G}-1-\mathrm{P})$

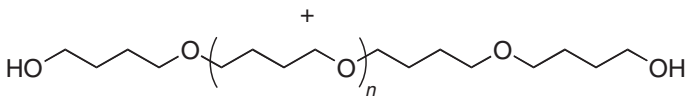

(a) PTHF-1K: $M_{\mathrm{n}}=1350, M_{\mathrm{w}} / M_{\mathrm{n}}=2.86$

PTHF $\left\{\right.$ (b) PTHF-3K: $M_{\mathrm{n}}=3040, M_{\mathrm{w}} / M_{\mathrm{n}}=3.13$

(c) PTHF-6K: $M_{\mathrm{n}}=6330, M_{\mathrm{w}} / M_{\mathrm{n}}=2.45$

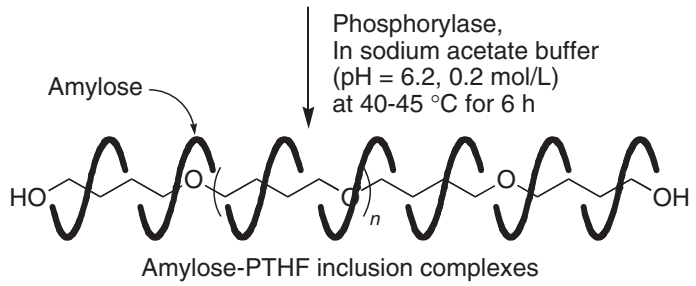

Scheme 1. Preparation of amylose-PTHF inclusion complexes by vinetwining polymerization.

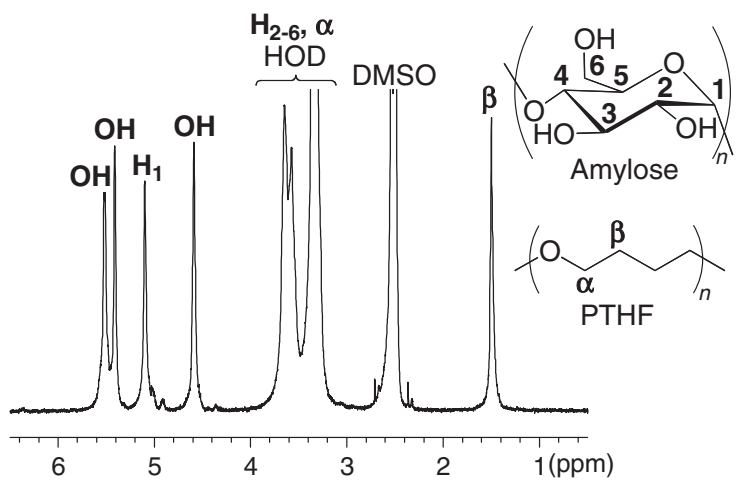

Figure 1. ${ }^{1} \mathrm{H}$ NMR spectrum of the product obtained by the vine-twining polymerization using PTHF-1K.

the signals not only due to amylose but also due to PTHF-1K (Figure 1), in spite of washing with acetone as the good solvent for PTHF-1K, indicating that PTHF-1K was included in the cavity of amylose. In addition, the XRD pattern of the product obtained from PTHF-1K showed two diffraction peaks at $2 \theta=c a .13^{\circ}$ and $20^{\circ}$ (Figure 2 ), which was similar to those of the inclusion complexes of amylose with monomeric compounds ${ }^{11}$ and with polymers. ${ }^{7}$ These ${ }^{1} \mathrm{H}$ NMR and XRD results indicate that the product is inclusion complex composed of amylose and PTHF. Similarly, the products obtained from PTHF-3K and PTHF-6K as the guest polymers were charac-

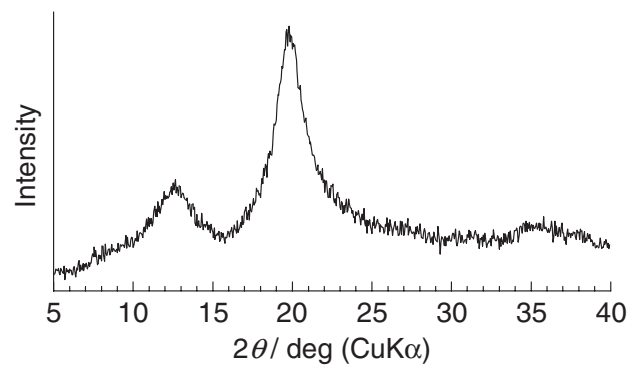

Figure 2. XRD pattern of the product obtained by the vine-twining polymerization using PTHF-1K.

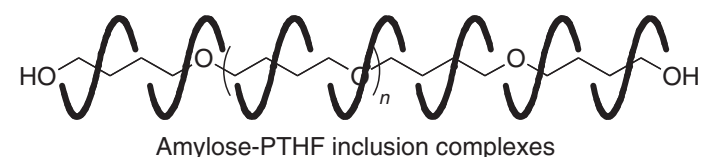

$$
\begin{aligned}
& \text { 1) Stirring in DMSO } \\
& \text { at } 40^{\circ} \mathrm{C} \text { for } 13 \mathrm{~h} \\
& \text { 2) Adding to excess } \\
& \text { amount of acetone }
\end{aligned}
$$

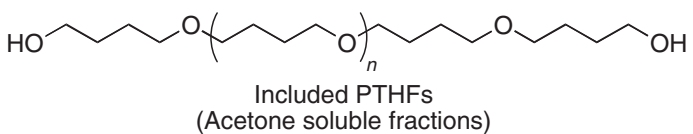

(Acetone soluble fractions)

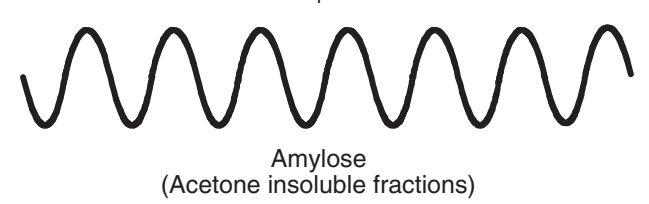

Scheme 2. Extraction of PTHFs from the inclusion complexes

terized by ${ }^{1} \mathrm{H}$ NMR and XRD measurements, which also supported the structures of the inclusion complexes.

To evaluate MWs and MWDs of PTHFs included in the cavity of amylose, PTHFs were extracted from the inclusion complexes according to the following operation. After the solutions of the inclusion complexes, obtained by each experiment, in DMSO were stirred at $40^{\circ} \mathrm{C}$ for $13 \mathrm{~h}$, they were added to an excess amount of acetone (Scheme 2). Then, the acetone soluble fractions were collected and characterized by ${ }^{1} \mathrm{H}$ NMR measurements to be PTHFs.

The $M_{\mathrm{n}}$ and $M_{\mathrm{w}} / M_{\mathrm{n}}$ of the extracted PTHFs were estimated by ${ }^{1} \mathrm{H}$ NMR and GPC measurements, respectively. When PTHF-3K with $M_{\mathrm{w}} / M_{\mathrm{n}}=3.13$ was employed as the guest polymer for the vine-twining polymerization, MWD of PTHF included in the cavity of amylose became narrow $\left(M_{\mathrm{w}} / M_{\mathrm{n}}=\right.$ 1.46) although its MW $\left(M_{\mathrm{n}}=3590\right)$ was almost same as that of the employed one $\left(M_{\mathrm{n}}=3040\right)$ (Figure $\left.3 \mathrm{~b}\right)$. These results indicate that amylose selectively included a specific range of MWs in PTHF-3K in the vine-twining polymerization. To investigate the effect of average MWs of the guest PTHFs on the inclusion behavior of amylose, we performed the vinetwining polymerization using PTHF-1K with $M_{\mathrm{w}} / M_{\mathrm{n}}=2.86$ and PTHF-6K with $M_{\mathrm{w}} / M_{\mathrm{n}}=2.45$ as the guest polymers. Consequently, the $M_{\mathrm{n}}$ and $M_{\mathrm{w}} / M_{\mathrm{n}}$ of PTHFs included in the 

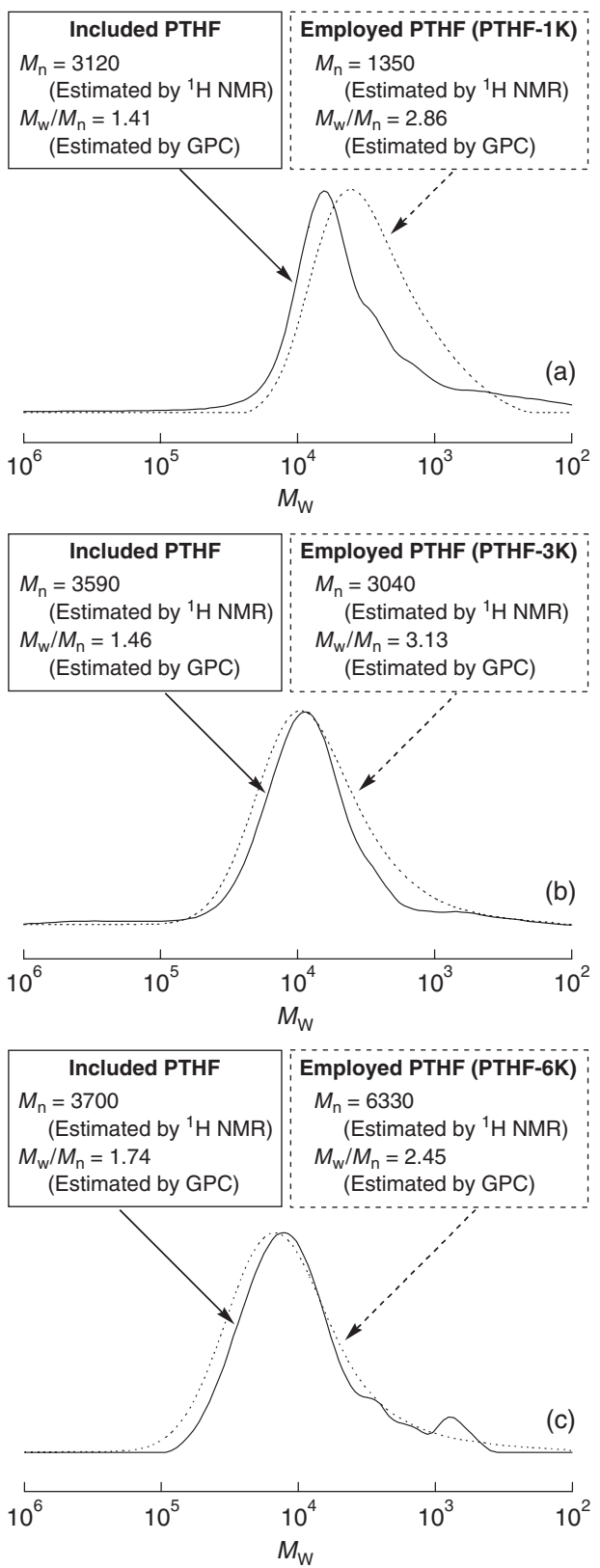

Figure 3. GPC curves of PTHFs employed as guests; (a) PTHF-1K, (b) PTHF-3K, and (c) PTHF-6K, and those of PTHFs included in the cavity of amylose in the vine-twining polymerization using (a) PTHF-1K, (b) PTHF-3K, and (c) PTHF-6K

cavity of amylose were almost same as those using PTHF-3K as described above (Figure $3 \mathrm{a}, \mathrm{c}$ ). Thus it can be concluded that, in this polymerization system, amylose selectively includes a specific range of MWs in PTHFs. These results are probably because of the difference in inclusion ability of amylose toward PTHFs with various MWs in the vine-twining polymerization.

\section{CONCLUSIONS}

In this paper, we described that amylose selectively included a specific range of MWs in guest PTHFs in the vine-twining polymerization. When the phosphorylase-catalyzed enzymatic polymerization of $\alpha$-D-glucose 1-phosphate from maltoheptaose as a primer was performed in the presence of PTHFs with different average MWs, amylose included almost same MW range with low distribution in any PTHFs used.

Acknowledgment. This work was supported in part by a fund from Sekisui Chemical Co., Ltd. and a Grant-in-Aid for Scientific Research from Ministry of Education, Culture, Sports, Science, and Technology, Japan (No. 19550126). We acknowledge the gift of phosphorylase from Ezaki Glico Co. Ltd., Osaka, Japan.

Received: April 27, 2009

Accepted: June 5, 2009

Published: July 23, 2009

\section{REFERENCES}

1. a) C. J. Pedersen, J. Am. Chem. Soc., 89, 7017 (1967).

b) G. W. Gokel, W. M. Leevy, and M. E. Weber, Chem. Rev., 104, 2723 (2004).

2. A. Harada, M. Okada, J. Li, and M. Kamachi, Macromolecules, 28, 8406 (1995).

3. M. Kowblansky, Macromolecules, 18, 1776 (1985).

4. a) O. K. Kim, L. S. Choi, H. Y. Zhang, X. H. He, and Y. H. Shih, J. Am. Chem. Soc., 118, 12220 (1996).

b) L. S. Choi and O. K. Kim, Macromolecules, 31, 9406 (1998).

c) T. Sanji, N. Kato, M. Kato, and M. Tanaka, Angew. Chem., Int. Ed., 44, 7301 (2005).

d) I. Lalush, H. Bar, I. Zakaria, S. Eichler, and E. Shimoni, Biomacromolecules, 6, 121 (2005).

e) T. Sanji, N. Kato, and M. Tanaka, Macromolecules, 39, 7508 (2006).

f) O. K. Kim, J. Je, and J. S. Melinger, J. Am. Chem. Soc., 128, 4532 (2006).

g) T. Sanji, N. Kato, and M. Tanaka, Org. Lett., 8, 235 (2006).

5. a) R. L. Shogren, R. V. Green, and Y. V. Wu, J. Appl. Polym. Sci., 42, 1701 (1991).

b) R. L. Shogren, Carbohydr. Polym., 22, 93 (1993).

c) A. Star, D. W. Steuerman, J. R. Heath, and J. F. Stoddart, Angew. Chem., Int. Ed., 41, 2508 (2002).

d) M. Ikeda, Y. Furusho, K. Okoshi, S. Tanahara, K. Maeda, S. Nishino, T. Mori, and E. Yashima, Angew. Chem., Int. Ed., 45, 6491 (2006).

e) T. Kida, T. Minabe, S. Okada, and M. Akashi, Chem. Commun., 1559 (2007).

f) M. J. Frampton, T. D. W. Claridge, G. Latini, S. Brovelli, F. Cacialli, and H. L. Anderson, Chem. Commun., 2797 (2008).

6. The enzymatic polymerization is a useful tool for the regio- and stereocontrolled preparation of polysaccharides:

a) S. Kobayashi, H. Uyama, and S. Kimura, Chem. Rev., 101, 3793 (2001).

b) S. Shoda, R. Izumi, and M. Fujita, Bull. Chem. Soc. Jpn., 76, 1 (2003).

c) S. Kobayashi, M. Ohmae, S. Fujikawa, and H. Ochiai, Macromol. Symp., 226, 147 (2005).

d) S. Kobayashi and M. Ohmae, Adv. Polym. Sci., 194, 159 (2006). For example, phosphorylase-catalyzed enzymatic polymerization using $\alpha$-D-glucose 1-phosphate (G-1-P) proceeds with the regioand stereoselective construction of an $\alpha$-glycosidic bond under mild conditions, leading to the direct formation of amylose in aqueous media. This polymerization is initiated from a maltooligosaccharide primer such as $G_{7}$. Then, the propagation proceeds through the 
following reversible reaction to produce a $(1 \rightarrow 4)$ - $\alpha$-glucan chain, i.e., amylose:

$$
[(\alpha, 1 \rightarrow 4)-\mathrm{G}]_{n}+\mathrm{G}-1-\mathrm{P} \leftrightarrows[(\alpha, 1 \rightarrow 4)-\mathrm{G}]_{n+1}+\mathrm{P}
$$

In the reaction, a glucose unit is transferred from G-1-P to the nonreducing 4-OH terminus of a $(1 \rightarrow 4)$ - $\alpha$-glucan chain, resulting in inorganic phosphate $(\mathrm{P})$ :

e) G. Ziegast and B. Pfannemüller, Carbohydr. Res., 160, 185 (1987).

7. a) J. Kadokawa, Y. Kaneko, H. Tagaya, and K. Chiba, Chem. Commun., 449 (2001).

b) J. Kadokawa, Y. Kaneko, A. Nakaya, and H. Tagaya, Macromolecules, 34, 6536 (2001).

c) J. Kadokawa, Y. Kaneko, S. Nagase, T. Takahashi, and H. Tagaya, Chem. Eur. J., 8, 3321 (2002).

d) J. Kadokawa, A. Nakaya, Y. Kaneko, and H. Tagaya, Macromol. Chem. Phys., 204, 1451 (2003).

e) Y. Kaneko and J. Kadokawa, Chem. Rec., 5, 36 (2005).

f) Y. Kaneko and J. Kadokawa, J. Biomater. Sci., Polym. Ed., 17, 1269 (2006). g) Y. Kaneko, K. Beppu, and J. Kadokawa, Biomacromolecules, 8, 2983 (2007).

h) Y. Kaneko, K. Beppu, and J. Kadokawa, Macromol. Chem. Phys., 209, 1037 (2008).

i) Y. Kaneko, Y. Saito, A. Nakaya, J. Kadokawa, and H. Tagaya, Macromolecules, 41, 5665 (2008).

j) Y. Kaneko, K. Beppu, T. Kyutoku, and J. Kadokawa, Polym. J., 41, 279 (2009).

8. M. Yanase, H. Takata, K. Fujii, T. Takaha, and T. Kuriki, Appl. Environ. Microbiol., 71, 5433 (2005).

9. S. Smith and A. J. Hubin, J. Macromol. Sci., Part A: Pure Appl. Chem., 7, 1399 (1973).

10. V. V. Braunmühl, G. Jonas, and R. Stadler, Macromolecules, 28, 17 (1995).

11. a) H. D. Seneviratne and C. G. Biliaderis, J. Cereal Sci., 13, 129 (1991).

b) N. Jeannette, S. Bettina, C. F. Béatrice, and E. Felix, Food Hydrocolloids, 11, 27 (1997). 